\title{
Finite-Time Synchronizing Control for Chaotic Neural Networks
}

\author{
Chao Zhang, Qiang Guo, and Jing Wang \\ National Engineering Research Center of Advanced Rolling, University of Science and Technology Beijing, Beijing 100083, China \\ Correspondence should be addressed to Qiang Guo; guoqiang@nercar.ustb.edu.cn
}

Received 11 April 2014; Revised 4 June 2014; Accepted 4 June 2014; Published 17 June 2014

Academic Editor: Hongli Dong

Copyright (C) 2014 Chao Zhang et al. This is an open access article distributed under the Creative Commons Attribution License, which permits unrestricted use, distribution, and reproduction in any medium, provided the original work is properly cited.

\begin{abstract}
This paper addresses the finite-time synchronizing problem for a class of chaotic neural networks. In a real communication network, parameters of the master system may be time-varying and the system may be perturbed by external disturbances. A simple highgain observer is designed to track all the nonlinearities, unknown system functions, and disturbances. Then, a dynamic active compensatory controller is proposed and by using the singular perturbation theory, the control method can guarantee the finitetime stability of the error system between the master system and the slave system. Finally, two illustrative examples are provided to show the effectiveness and applicability of the proposed scheme.
\end{abstract}

\section{Introduction}

The chaotic system exhibits unpredictable and irregular dynamics which has been found in many practical systems. Interestingly, small differences in the initial state can lead to significant differences in the chaotic system state. Chaotic synchronization is receiving increasing attention within the area of nonlinear dynamics [1]. One of the most important applications is secure communication [2], where an information-bearing signal is hidden on a chaotic carrier signal. Synchronization of chaotic systems has been thought possible since the seminal work of Pecora and Carroll. They proposed a drive-response concept for constructing synchronization of coupled chaotic systems [3]. State variables of a given chaotic master system are used as inputs to drive a slave system that is a duplicated one of the drive system. Under some conditions, the state evolution of the slave system is shown to synchronize with that of the master system. There are many important results focusing on many typical chaotic systems such as the Chua system, Lorenz system, and Lur'e system (see, e.g., [3-7] and references therein).

On the other hand, neural networks have been extensively studied over the past two decades for their potential applications in modeling complex dynamics, nonlinear programming, image processing, pattern recognition, associative memory, and so forth [8]. Recently, it has been shown that if the networks parameters and time delays are appropriately chosen, these networks can exhibit some complicated dynamics and even chaotic behaviors [9-11]. Therefore, the synchronization of chaotic neural networks has become an important area of study as other kinds of chaotic systems. In this field, various synchronization schemes have been proposed (see, e.g., [12-28]). In [12,13], synchronization of delayed neural networks is realized by using adaptive control and parameters identification. For time-varying networks, the property of synchronization is discussed in [14, 15]. Adaptive synchronization of two different chaotic neural networks is realized by using Lyapunov method and linear matrix inequalities in [16]. Periodically intermittent control is used to solve the problem of exponential synchronization of stochastic Cohen-Grossberg neural networks [18].

In real physical systems, how to design the systems for special signal transmission channels has become a critical issue. Secure communication has to rely on common communication networks in many applications. Because of the network communication introduced [19], signal transmission channels often involve data missing, system varying, and environment disturbances [20-23]. These lead to a limitation of some existing control schemes when they are applied to a practical environment since they are based on the accurate master-slave system parameters. However, there are few studies on the synchronization issue for this class of chaotic neural 
networks with unknown parameters and disturbances [2429]. Most of them focus on either the unknown parameters or the disturbance, but not both.In addition, some of them take the disturbances as a kind of special stochastic structure $[25,26]$.

Moreover, in real applications, we not only need the systems to guarantee synchronization but also need them to synchronize in the shortest possible time. If the slave system synchronizes with the master system in infinite time, the system will not be able to work effectively. In view of all of the above-mentioned facts, the aim of this paper is to investigate the finite-time synchronization for a class of delayed neural networks with all the uncertainty and external disturbances.

In this paper, an active control method is proposed to control the slave system based on the terminal attractor for the purpose of guaranteeing that the error system between the master system and the slave system will achieve finitetime stability. In the meantime, in order to realize the controller, an effective state observer based on a special tracking differentiator is designed to estimate all the uncertainties and disturbances in a very short time. The approximate finitetime stability of the closed-loop system is analysed in detail on the basis of the singular perturbation theory. Moreover, the results are applied to some chaotic neural networks. Two numerical simulations are also given to validate the effectiveness of the proposed control strategy on the Matlab TrueTime simulation platform.

The rest of this paper is organized as follows. In Section 2, the problem formulations are presented after some preliminaries are given. In Section 3, the observer and the controller are proposed to realize the finite-time synchronization. Numerical simulations are given to demonstrate and also visualize the effectiveness of the proposed theoretical results in Section 4. Finally, some concluding remarks are drawn in Section 5 .

\section{Problem Formulation and Some Preliminaries}

In this section, we formulate an error system for the masterslave synchronization of delayed neural networks. Some preliminary knowledge will be presented for the derivation of our main results. Consider a class of delayed neural network models consisting of $N$ identical nodes, which can be described by the following differential equation:

$$
\begin{aligned}
\dot{x}_{i}(t)= & -c_{i} x_{i}(t)+\sum_{j=1}^{n} a_{i j} f_{j}\left(x_{j}(t)\right) \\
& +\sum_{j=1}^{n} b_{i j} f_{j}\left(x_{j}(t-\tau(t))\right),
\end{aligned}
$$

or in a compact form as follows:

$$
\dot{x}(t)=-C x(t)+A f(x(t))+B f(x(t-\tau(t))),
$$

where $x(t)=\left[x_{1}(t), x_{2}(t), \ldots, x_{i}(t), \ldots, x_{n}(t)\right]^{T} \in \mathbb{R}^{n}$ is the state vector associated with the $n$ neurons, $C=$ $\operatorname{diag}\left\{c_{1}, c_{2}, \ldots, c_{n}\right\}$ is a positive diagonal matrix, denoting the rate with which the cell $i$ resets its potential to the resting state when isolated from other cells and inputs, and $A=$ $\left(a_{i j}\right)_{n \times n}$ and $B=\left(b_{i j}\right)_{n \times n}$ are the connection weight matrix and the delayed connection weight matrix. $f_{j}(\cdot)(j=1,2, \ldots, n)$ stands for activation functions, so $f_{i}\left(x_{j}(t)\right)$ is the output of the $j$ th neuron of the network and $\tau(t)$ are time-varying time delays of the neural network. The initial conditions are given by $x_{i}(t)=\phi_{x i}(s) \in C([-\tau, 0], \mathbb{R})$, where $C([-\tau, 0], \mathbb{R})$ denotes the set of all continuous functions from $[-\tau, 0]$ to $\mathbb{R}$.

The corresponding slave system is established as follows:

$$
\begin{aligned}
\dot{y}_{i}(t)= & -\bar{c}_{i} y_{i}(t)+\sum_{j=1}^{n} \bar{a}_{i j} f_{j}\left(y_{j}(t)\right) \\
& +\sum_{j=1}^{n} \bar{b}_{i j} f_{j}\left(y_{j}(t-\tau(t))\right)+u_{i}(t),
\end{aligned}
$$

where $y(t)=\left[y_{1}(t), y_{2}(t), \ldots, y_{i}(t), \ldots, y_{n}(t)\right]^{T} \in \mathbb{R}^{n}$ is the neuron state of the slave system. All the system parameters $\bar{C}=\operatorname{diag}\left(\bar{c}_{1}, \bar{c}_{2}, \ldots, \bar{c}_{n}\right), \bar{A}=\left(\bar{a}_{i j}\right)_{n \times n}$, and $\bar{B}=\left(\bar{b}_{i j}\right)_{n \times n}$ have the same definitions as in (1) but may not be equal to parameters of the master system all the time. $u_{i}(t)(i=$ $1,2, \ldots, n)$ is the control signals and the initial conditions are given by $y_{i}(t)=\varphi_{y i}(s) \in C([-\tau, 0], \mathbb{R})$.

In network-based synchronization systems, there exist system uncertainties and external disturbances when network communications are introduced. In this paper, we assume that all the parameters of the master system are uncertain or even time-varying to represent the system's internal uncertainty. For the external disturbance, it is inevitable in physics systems and it plays an important role in chaos synchronization in different ways [25]. Some papers regard a type of Brownian motions as a result of the occurrence of random uncertainties in the communication channels in the error system between the master system and the slave system. In this paper we do not care about the specific form of the disturbance, so we regard all the input disturbances and other uncertain nonlinearities as a more general form $H(t)$. $H(t)=\left[h_{1}(t), h_{2}(t), \ldots, h_{n}(t)\right]^{T}$ is unknown and bounded; that is, $\left\|H_{i}(t)\right\| \leq D$ and $D$ is an unknown positive number. For simplicity, the master system is supposed to be without disturbances and disturbance dynamics are exhibited in the slave system.

In order to give a better explanation, subtract the master system (1) from the slave system (3) and define the synchronization errors as $e_{i}(t)=y_{i}(t)-x_{i}(t)$. Consider the input disturbance $H(t)$, writing the error system in an expanded form as follows:

$$
\begin{aligned}
\dot{e}_{i}(t)= & -c_{i} e_{i}(t)-\left(\bar{c}_{i}-c_{i}\right) y_{i}(t)+\sum_{j=1}^{n} a_{i j} g_{j}\left(e_{j}(t)\right) \\
& +\sum_{j=1}^{n}\left(\bar{a}_{i j}-a_{i j}\right) f_{j}\left(y_{j}(t)\right)+\sum_{j=1}^{n} b_{i j} g_{j}\left(e_{j}(t-\tau(t))\right)
\end{aligned}
$$




$$
\begin{aligned}
& +\sum_{j=1}^{n}\left(\bar{b}_{i j}-b_{i j}\right) f_{j}\left(y_{j}(t-\tau(t))\right)+u_{i}(t)+h_{i}(t) e_{i}(s) \\
= & \varphi_{i}(s)-\phi_{i}(s), \quad s \in(-\tau, 0],
\end{aligned}
$$

where $g_{j}\left(e_{j}(t)\right)=f_{j}\left(e_{j}(t)+x_{j}(t)\right)-f_{j}\left(x_{j}(t)\right)$. Then, our goal is to design the controller $u_{i}(t)(i=1,2, \ldots, n)$ which makes the dynamical system (4) stabilized in a finite-time.

To facilitate further work, it is assumed that some reasonable conditions are satisfied.

(H1) The activation functions $f_{i}(\cdot)(j=1,2, \ldots, n)$ satisfy the Lipschitz condition with a positive $L_{i}$ :

$$
\left\|f_{i}\left(x_{1}\right)-f_{i}\left(x_{2}\right)\right\| \leq L_{i}\left\|x_{1}-x_{2}\right\|, \quad \forall x_{1}, x_{2} \in R .
$$

(H2) The time delay $\tau(t)$ is a bounded and differentiable function satisfying $0 \leq \tau(t)<\infty$ and $\dot{\tau}(t)<\infty$.

(H3) The unknown nonlinear disturbance $h_{i}(t)$ is a differentiable function satisfying $\dot{h}(t)<\infty$ and $0 \leq h_{i}(t)<$ $\infty$.

\section{Main Results}

In this section, we introduce the terminal attractor and its work principle first, and then we give the design of the controller and the observer. Finally, some criteria are derived to ensure finite-time synchronization between the master and slave systems.

In system (4), we define

$$
\begin{aligned}
\eta_{i}= & -c_{i} e_{i}(t)-\left(\bar{c}_{i}-c_{i}\right) y_{i}(t)+\sum_{j=1}^{n} a_{i j} g_{j}\left(e_{j}(t)\right) \\
& +\sum_{j=1}^{n}\left(\bar{a}_{i j}-a_{i j}\right) f_{j}\left(y_{j}(t)\right)+\sum_{j=1}^{n} b_{i j} g_{j}\left(e_{j}(t-\tau(t))\right) \\
& +\sum_{j=1}^{n}\left(\bar{b}_{i j}-b_{i j}\right) f_{j}\left(y_{j}(t-\tau(t))\right)+h_{i}
\end{aligned}
$$

and rewrite the error dynamical system (4) as follows:

$$
\begin{gathered}
\dot{e}_{1}(t)=\eta_{1}+u_{1} \\
\dot{e}_{2}(t)=\eta_{2}+u_{2} \\
\vdots \\
\dot{e}_{n}(t)=\eta_{n}+u_{n} .
\end{gathered}
$$

Obviously, $\boldsymbol{\eta}=\left[\eta_{1}, \ldots, \eta_{i}, \ldots, \eta_{n}\right]^{T}$ denotes the overall uncertainty of all the unknown parameters and disturbances. Considering the form of $\boldsymbol{\eta}$ and assumptions in the previous section, we obtain that $\boldsymbol{\eta}$ is differentiable and satisfies $0 \leq$ $\dot{\eta}_{i}(t)<\infty$.
3.1. Terminal Attractor. Terminal attractor can realize finitetime stability of the system using the non-Lipschitz continuity of equilibrium points [30]. Consider a differential equation as follows:

$$
\dot{\rho}=-\beta \rho^{q / p}
$$

where $q$ and $p$ are positive odd numbers. Obviously, $\rho=0$ is the equilibrium point and we can obtain $\lim _{\rho \rightarrow 0} d \dot{\rho}=\infty$. If $\rho=0, d \dot{\rho}$ does not satisfy Lipschitz continuity and this leads to the finite-time stability of (8). Meanwhile, $d \dot{\rho} \rightarrow$ $-\infty(\rho \rightarrow 0)$, and we can calculate the Jacobian matrix of (8). Consider

$$
\lim _{\rho \rightarrow 0} \frac{d \dot{\rho}}{d \rho}=-\infty
$$

In other words, if $\rho$ approaches the equilibrium point $\rho=0$, its eigenvalue will approach $-\infty$. The trajectory of the system will converge to the equilibrium point at an infinite rate and this leads to its finite-time stability. On the other hand, we solve the equation directly, and the solutions all satisfy (10) for arbitrary initial states $\rho(0)$. Consider

$$
\frac{p}{p-q}\left[\rho\left(t_{s}\right)^{(p-q) / p}-\rho(0)^{(p-q) / p}\right]=-\beta t_{s} .
$$

From (10), we can obtain $t_{s}=(p / \beta(p-q))|\rho(0)|^{(p-q) / p}$, and $t_{s}$ is the required time from $\rho(0)$ to $\rho=0$.

3.2. Controller and Observer Design. If $\boldsymbol{\eta}=\left[\eta_{1}, \ldots, \eta_{i}\right.$, $\left.\ldots, \eta_{n}\right]^{T}$ is previously identified, then we can design the active compensatory controller based on the terminal attractor. Consider

$$
\begin{gathered}
u_{1}=-k_{1} e_{1}^{\alpha}-\eta_{1} \\
u_{2}=-k_{2} e_{2}^{\alpha}-\eta_{2} \\
\vdots \\
u_{i}=-k_{i} e_{i}^{\alpha}-\eta_{i} \\
\vdots \\
u_{n}=-k_{n} e_{n}^{\alpha}-\eta_{n},
\end{gathered}
$$

where $k_{1}, k_{2}, \ldots, k_{n}>0$, is the feedback strength and $\alpha=q / p$, with $q, p$ being positive odd numbers and $q<p$.

Substitute (11) into the error dynamical system (7); a new form of the system is shown in (12). It is easy to check the 
finite-time stability of this closed-loop system according to terminal attractor. Consider

$$
\begin{gathered}
\dot{e}_{1}(t)=-k_{1} e_{1}^{\alpha} \\
\dot{e}_{2}(t)=-k_{2} e_{2}^{\alpha} \\
\vdots \\
\dot{e}_{i}(t)=-k_{i} e_{i}^{\alpha} \\
\vdots \\
\dot{e}_{n}(t)=-k_{n} e_{n}^{\alpha} .
\end{gathered}
$$

In practice, the uncertain nonlinear term $\eta_{i}$ is totally unknown, so we cannot realize the synchronization by (11). We need to achieve its realistic form.

To estimate $f_{i}$, based on [31-33], here we introduce a differential observer (13). Using a similar method mentioned in $[31,34]$, it is easy to prove that the observer can track system uncertainties in a short time. The time needed is $O(\varepsilon)$. Consider

$$
\begin{aligned}
& \dot{w}_{i}=-\tilde{u}_{i}-\frac{\lambda}{\varepsilon^{2}}\left(w_{i}+e_{i}\right), \\
& \tilde{\eta}_{i}=\frac{\lambda}{\varepsilon^{2}}\left(w_{i}+e_{i}\right),
\end{aligned}
$$

where $i=1,2, \ldots, n, w_{i}(0)=-e_{i}(0)$, and $\tilde{\eta}_{i}=0, \tilde{\eta}_{i}$ is the estimation value of $\eta_{i}, \lambda>0$, and $0<\varepsilon \ll 1$. Therefore, we can obtain the realistic form of $u_{i}$. Consider

$$
\begin{gathered}
\tilde{u}_{1}=-k_{1} e_{1}^{\alpha}-\tilde{\eta}_{1} \\
\tilde{u}_{2}=-k_{2} e_{2}^{\alpha}-\widetilde{\eta}_{2} \\
\vdots \\
\tilde{u}_{i}=-k_{i} e_{i}^{\alpha}-\tilde{\eta}_{i} \\
\vdots \\
\widetilde{u}_{n}=-k_{n} e_{n}^{\alpha}-\widetilde{\eta}_{n} .
\end{gathered}
$$

But peaking phenomenon is an important feature of the observer and in particular the controller may destabilize the closed-loop system as the observer gain is driven sufficiently high [34]. So we design the control as a globally bounded function so that it is saturated during the peaking period. Define $m_{i}$ as the upper bound of all input control $\widetilde{u}_{i}$ that can stabilize the closed-loop system. Consider

$$
\widetilde{u}_{i}=\operatorname{Sat}\left(\widetilde{u}_{i}\right)= \begin{cases}m_{i} \cdot \operatorname{sign}\left(\tilde{u}_{i}\right) & \left|\tilde{u}_{i}\right|>m_{i}, \\ \widetilde{u}_{i} & \left|\tilde{u}_{i}\right| \leq m_{i} .\end{cases}
$$

3.3. Analysis of the Finite-Time Synchronization. Define the estimate error as $\delta_{i}=\eta_{i}-\widetilde{\eta}_{i}$, substitute controller (14) into system (11), and rewrite the error dynamical system as

$$
\begin{gathered}
\dot{e}_{1}(t)=-k_{1} e_{1}^{\alpha}+\delta_{1} \\
\dot{e}_{2}(t)=-k_{2} e_{2}^{\alpha}+\delta_{2} \\
\vdots \\
\dot{e}_{i}(t)=-k_{i} e_{i}^{\alpha}+\delta_{i} \\
\vdots \\
\dot{e}_{n}(t)=-k_{n} e_{n}^{\alpha}+\delta_{n}
\end{gathered}
$$

or in a compact form as $\dot{\mathbf{e}}=\mathbf{F}(\mathbf{e})+\boldsymbol{\delta}$, where $F_{i}\left(e_{i}\right)=-k_{i} e_{i}^{\alpha}$. From (13), we obtain $\delta_{i}=-\left(\lambda / \varepsilon^{2}\right) \delta_{i}+\dot{\eta}_{i}$ or in a compact form $\varepsilon^{2} \dot{\boldsymbol{\delta}}=\mathrm{A} \boldsymbol{\delta}-\varepsilon^{2} \dot{\boldsymbol{\eta}}$, where $n \times n$ matrix $\mathrm{A}=\operatorname{diag}\{-\lambda, \ldots,-\lambda\}$.

So we can describe the error dynamical system as

$$
\begin{aligned}
& \dot{\mathbf{e}}=\mathbf{F}(\mathbf{e})+\boldsymbol{\delta}, \\
& \varepsilon^{2} \dot{\boldsymbol{\delta}}=\mathrm{A} \boldsymbol{\delta}-\varepsilon^{2} \dot{\boldsymbol{\eta}} .
\end{aligned}
$$

When $\varepsilon$ is small enough, system (17) will be a singular perturbation system. The stability of this system is determined by its two subsystems [35]. When $\varepsilon=0$, equation $\mathrm{A} \boldsymbol{\delta}=\mathbf{0}$ has an only solution $\boldsymbol{\delta}=\mathbf{0}$. Based on the singular perturbation theory, the slow subsystem of the closed-loop system (16) is

$$
\dot{\mathbf{e}}=\mathbf{F}(\mathbf{e})
$$

and the fast subsystem is

$$
\dot{\zeta}=\mathrm{A} \zeta
$$

where $\dot{\boldsymbol{\zeta}}=d \boldsymbol{\delta} / d \sigma$ and $\sigma=t / \varepsilon^{2}$.

Lemma 1 (see [36]). Consider system $\dot{\mathbf{x}}=f(\mathbf{x})$, where $\mathbf{x} \in \mathbb{R}^{n}$, $f(\mathbf{x}): \mathbb{R}^{n} \rightarrow \mathbb{R}$, is a continuous function vector. The system is finite-time stable if and only if there exists a Lyapunov function $V(\mathbf{x})$ which satisfies the following inequality (20) for the system:

$$
\dot{V}(\mathbf{x})=\frac{\partial V}{\partial x} f(\mathbf{x}) \leq-\beta_{1}(V(\mathbf{x}))^{\beta_{2}},
$$

where $\beta_{1}>0$ and $\beta_{2} \in(0,1)$.

Theorem 2. If there exists a small positive $\varepsilon^{*}$, when $\varepsilon<\varepsilon^{*}$, the controller (14) based on the observer (13) makes the error system (17) finite-time stable and, thus, master system (1) and slave system (3) are finite-time synchronized.

Proof. For the subsystem (19), because A is a positive definite diagonal matrix, this system is exponentially stable, and it is easy to check its stability using Lyapunov function $W(\boldsymbol{\delta})=$ $\boldsymbol{\delta}^{\mathrm{T}} \boldsymbol{\delta}$, differentiating $W(\boldsymbol{\delta})$ with respect to (17). Consider

$$
\dot{W}=-\frac{2 \lambda}{\varepsilon^{2}} \boldsymbol{\delta}^{\mathrm{T}} \boldsymbol{\delta}+2 \boldsymbol{\delta}^{\mathrm{T}} \dot{\boldsymbol{\eta}} \leq-\frac{2 \lambda}{\varepsilon^{2}} W+2\|\dot{\boldsymbol{\eta}}\| \sqrt{W} .
$$




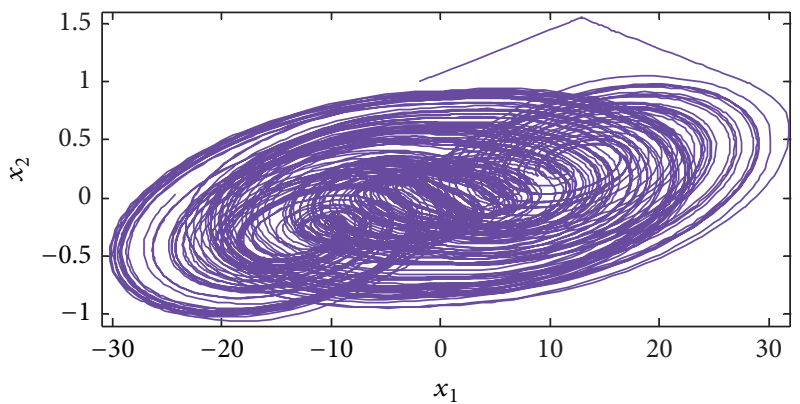

Figure 1: The phase plot of the master system (34).

Define $k_{0}=\sup \{\|\dot{\boldsymbol{\eta}}\|\}$ and $c_{1}=4 k_{0}^{2} / \lambda^{2}$. If $W(\boldsymbol{\delta}) \geq c_{1} \varepsilon^{4}$, (21) will change to

$$
\dot{W} \leq-\frac{\lambda}{\varepsilon^{2}} W
$$

Define the initial time as $t_{0}$ and make $c_{2}=W\left(\boldsymbol{\delta}\left(t_{0}\right)\right)$. When $c_{2} \geq c_{1} \varepsilon^{4}$, there exists a positive constant $\tau_{\varepsilon}$ for system (17) and $W(\boldsymbol{\delta})$ will satisfy (24). Consider

$$
\begin{gathered}
\tau_{\varepsilon}=-\frac{\varepsilon^{2}}{\lambda} \ln \left(\frac{c_{1} \varepsilon^{4}}{c_{2}}\right), \\
W(\delta(t)) \leq c_{2} e^{-\left(\lambda / \varepsilon^{2}\right)\left(t-t_{0}\right)} \quad t \in\left(t_{0}, t_{1}\right] \\
W\left(\delta\left(t_{1}\right)\right) \leq c_{1} \varepsilon^{4},
\end{gathered}
$$

where $t_{1}=t_{0}+\tau_{\varepsilon}$. When $W(\boldsymbol{\delta})=c_{1} \varepsilon^{4}$, (21) can still hold. So we obtain

$$
W(\boldsymbol{\delta})<c_{1} \varepsilon^{4}\left(t>t_{1}\right) .
$$

In other words, $W(\boldsymbol{\delta})$ is $O(\varepsilon)$. According to the definition of this Lyapunov function, we can get

$$
\|\boldsymbol{\delta}\| \leq c_{3} \varepsilon^{2} \quad\left(t>t_{1}\right),
$$

where $c_{3}=\sqrt{c_{1}}$. Equation (26) tells us that $\|\boldsymbol{\delta}\|$ is also $O(\varepsilon)$ when $t>t_{1}$.

For the order-reduced system (18), it is easy to explain its finite-time stability using the terminal attractor theory [30]. So, according to Lemma 1, there exists a Lyapunov function $V(\mathbf{e})$ that satisfies $V(0)=0$ and for $\forall \mathbf{e} \neq 0$ :

$$
\dot{V}(\mathbf{e})=\frac{\partial V}{\partial e} \dot{\mathbf{e}}=\frac{\partial V}{\partial \mathbf{e}} \mathbf{F}(\mathbf{e}) \leq-\beta_{1} V^{\beta_{2}},
$$

where $\beta_{1}>0$ and $0<\beta_{2}<1$.

On the other hand, we differentiate $V(\mathbf{e})$ with respect to the system (17). Consider

$$
\dot{V}(\mathbf{e})=\frac{\partial V}{\partial \mathbf{e}} \mathbf{F}(\mathbf{e})+\frac{\partial V}{\partial \mathbf{e}} \boldsymbol{\delta} \leq-\beta_{1} V^{\beta_{2}}+\frac{\partial V}{\partial \mathbf{e}} \boldsymbol{\delta} .
$$

Because the error $\mathbf{e}$ is bounded, we can get that $\partial V / \partial \mathbf{e}$ is bounded and define $M$ as the bound. When $t \rightarrow \infty$, consider (26) and then we have

$$
\dot{V}(\mathbf{e})=\leq-\beta_{1} V^{\beta_{2}}+\frac{\partial V}{\partial \mathbf{e}} \boldsymbol{\delta} \leq-\beta_{1} V^{\beta_{2}}+c_{3} M \varepsilon^{2} .
$$

If $V(\mathbf{e}) \geq c_{4} \varepsilon^{2 / \beta_{2}}\left(c_{4}=\left(2 c_{3} M / \beta_{1}\right)^{1 / \beta_{2}}\right)$ and $t>t_{1}$, (28) will change to

$$
\dot{V}(\mathbf{e}) \leq-\frac{\beta_{1}}{2} V^{\beta_{2}} .
$$

If $V\left(\mathbf{e}\left(t_{1}\right)\right) \geq c_{4} \varepsilon^{2 / \beta_{2}}$, there exists a time constant $T$ which makes (31) hold according to Lemma 1. Consider

$$
V\left(\mathbf{e}\left(t_{2}\right)\right) \leq c_{4} \varepsilon^{2 / \beta_{2}} \quad t_{2}=t_{1}+T .
$$

Calculate the integral of (29) from $V\left(\mathbf{e}\left(t_{1}\right)\right)$ to $c_{4} \varepsilon^{2 / \beta_{2}}$, and we can obtain

$$
T \leq \frac{2}{\beta_{1}\left(1-\beta_{2}\right)}\left(\left(V\left(\mathbf{e}\left(t_{1}\right)\right)\right)^{1-\beta_{2}}-\left(c_{4} \varepsilon^{2 / \beta_{2}}\right)^{1-\beta_{2}}\right) .
$$

When $V(\mathbf{e})=c_{4} \varepsilon^{2 / \beta_{2}}\left(t>t_{1}\right),(29)$ can still hold and $\dot{V}(\mathbf{e})<0$. So we obtain

$$
V(\mathbf{e})<c_{4} \varepsilon^{2 / \beta_{2}} \quad\left(t>t_{2}\right) .
$$

From (32) and $\beta_{2} \in(0,1)$, we can obtain that $V(\mathbf{e})$ is $O(\varepsilon)$ when $t>t_{2}$. In other words, for arbitrary small positive constant $r$, there exists a constant $\varepsilon^{*}$. Any $\varepsilon<\varepsilon^{*}$ will make the states of the closed-loop system (16) satisfy $\mathbf{e} \in B_{r}(0)$, where $\lim _{\mathcal{E}^{*} \rightarrow 0} r=0$ and $B_{r}(0)$ is a closed sphere whose centre is $\mathbf{e} \in B_{r}(0)$ and radius is $r$.

Meanwhile, according to the analysis above, the closedloop system (17) is finite-time stable and the time it needs to achieve stability is $\tau_{\varepsilon}+T$. This also means that the master system and the slave system are synchronized.

The proof is complete.

\section{Illustrative Examples}

In this section, two examples are presented below to illustrate the effectiveness of the previous results.

Example 3. Consider a typical two-dimensional chaotic neural network with mixed delay to be the master system (1) with the following parameters:

$$
\begin{gathered}
C=\left[\begin{array}{ll}
1 & 0 \\
0 & 1
\end{array}\right], \quad A=\left[\begin{array}{cc}
1+\frac{\pi}{4} & 20 \\
0.1 & 1+\frac{\pi}{4}
\end{array}\right], \\
B=\left[\begin{array}{cc}
-\frac{1.3 \sqrt{2} \pi}{4} & 0.1 \\
0.1 & -\frac{1.3 \sqrt{2} \pi}{4}
\end{array}\right], \\
f(s)=\frac{1}{2}(|s+1|-|s-1|), \quad \tau(t)=1 .
\end{gathered}
$$

It is investigated in [29] that the system possesses a chaotic behaviour. Figure 1 shows the chaotic behaviour of the system with the initial condition $\left[x_{1}(t), x_{2}(t)\right]=[0,1](t \in[-1,0])$. The slave system is given by (3) with parameters given by (34). Assume that there is an overall disturbance $\mathbf{h}(t)=0.01 \sin t$ in 


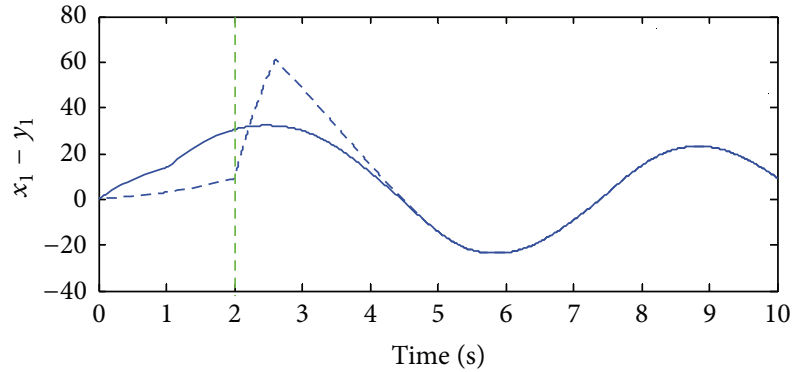

$\begin{array}{rr}- & x_{1} \\ --- & y_{1}\end{array}$

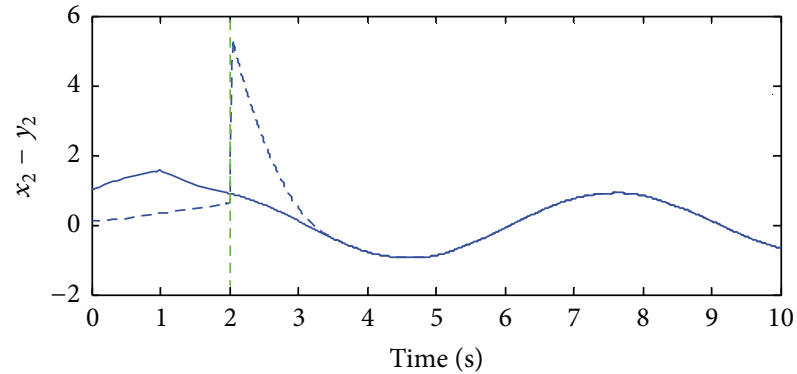

$-x_{2}$

(a)

(b)

FIGURE 2: State trajectories of slave system and master system.

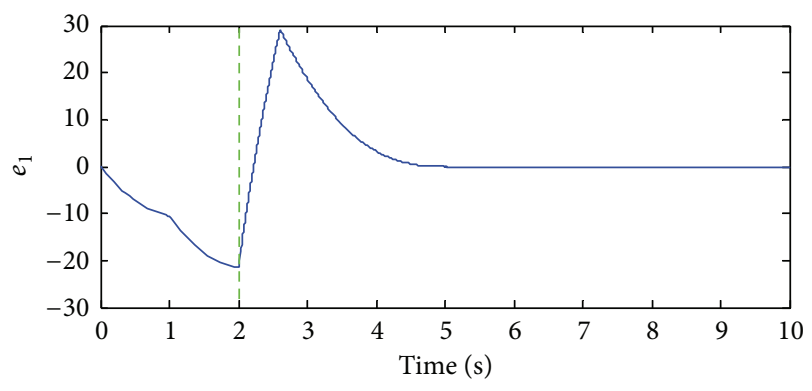

(a)

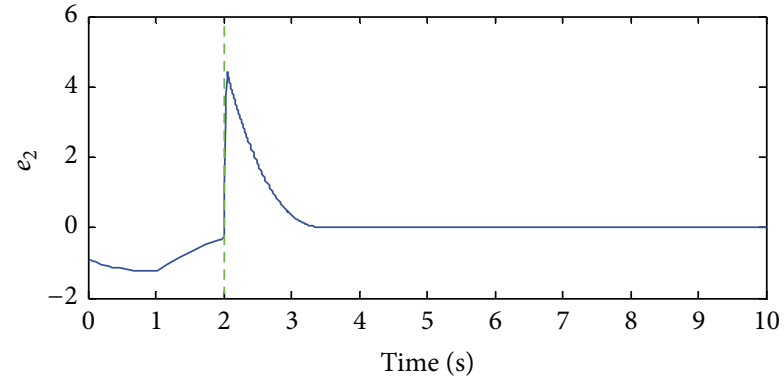

(b)

FIGURE 3: Synchronization errors of slave and master systems.

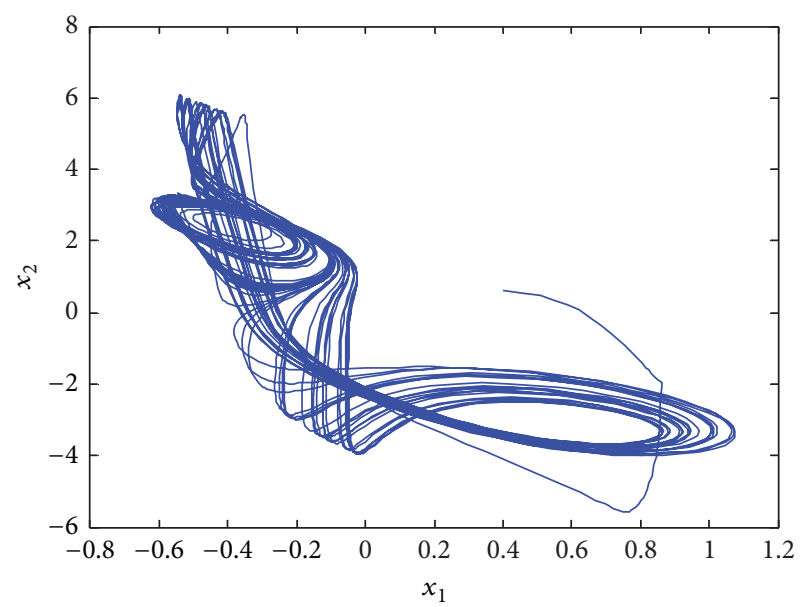

Figure 4: The phase plot of the master system (36).

the communication channels. The observer and the controller are designed as in (14) and (13), and the parameters are given as follows:

$$
\begin{gathered}
\lambda=8, \quad \varepsilon=0.05, \quad \alpha=\frac{3}{5} \\
k_{1}=4, \quad k_{2}=3 .
\end{gathered}
$$

Now we begin to simulate the synchronization in Matlab TrueTime simulation platform. The initial condition associated with the slave system is $[0.1,0.1]$. The simulation time is $15 \mathrm{~s}$, and the control signal begins to work at $5 \mathrm{~s}$. Figure 2 shows the trajectories of the master system and the slave system, and the dynamics of synchronization errors are illustrated in Figure 3. It can be seen that the error system is stabilized in a short time and the slave system indeed synchronizes with the master system.

Remark 4. There are some nondifferentiable points in $\boldsymbol{\eta}$ of this system because of the form of $f(s)$. However, we can see the effectiveness and applicability of the proposed method from these simulation results. That is to say, this control scheme has a great potential for complex systems.

Example 5. Consider the neural network (1) with the following parameters:

$$
\begin{gathered}
C=\left[\begin{array}{ll}
1 & 0 \\
0 & 1
\end{array}\right], \quad A=\left[\begin{array}{cc}
2 & -0.1 \\
-5 & 4.5
\end{array}\right], \\
B=\left[\begin{array}{cc}
-1.5 & -0.1 \\
-0.2 & -4
\end{array}\right],
\end{gathered}
$$

and $f(s)=\tanh (s)$.

The chaotic dynamic behaviours of this system have been studied in [37]. In the case of a time-varying time delay, let 


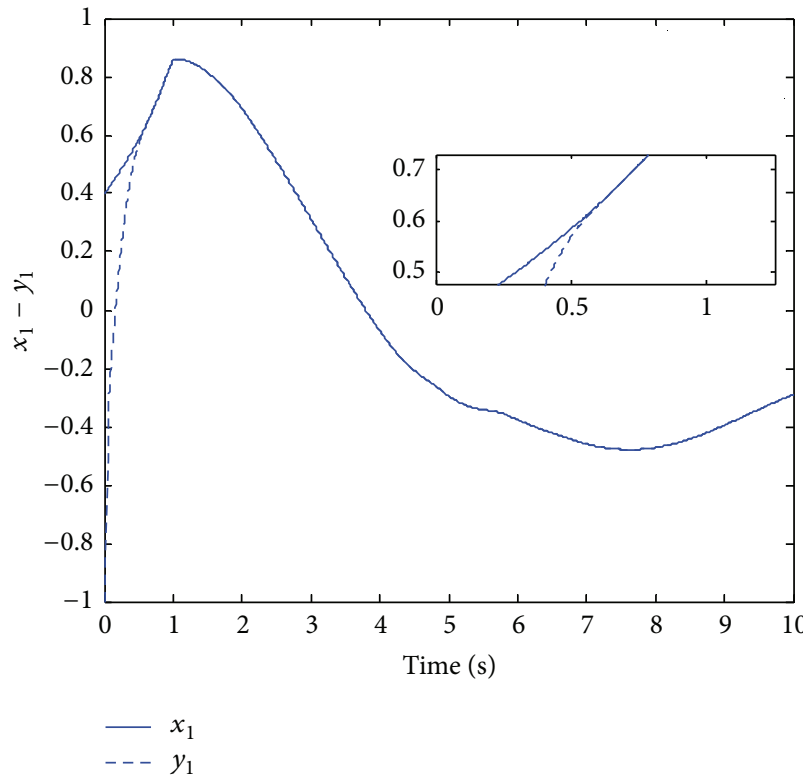

(a)

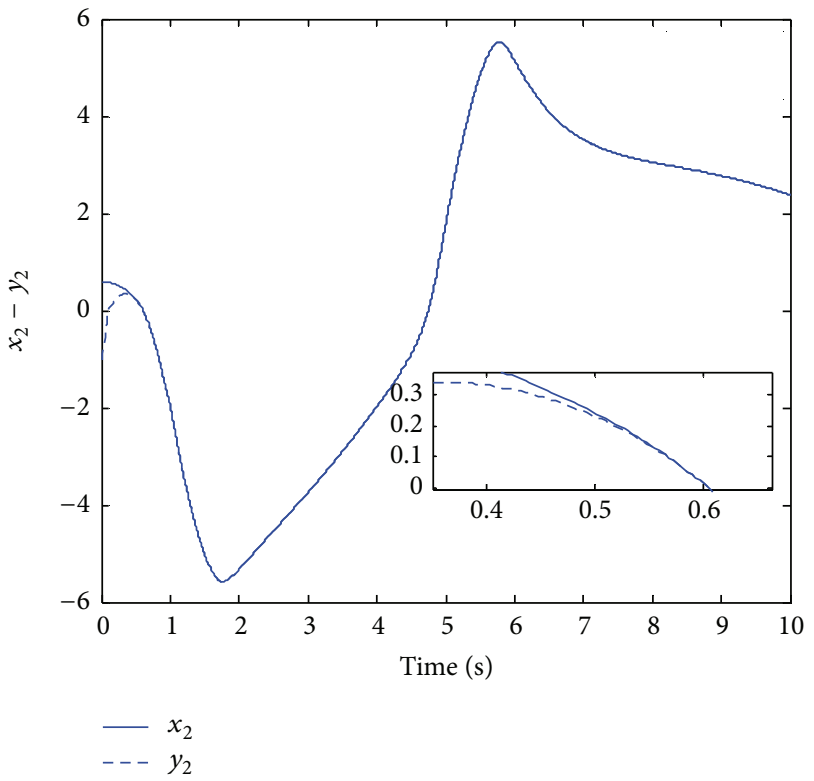

(b)

Figure 5: State trajectories of system (36) and system (37).

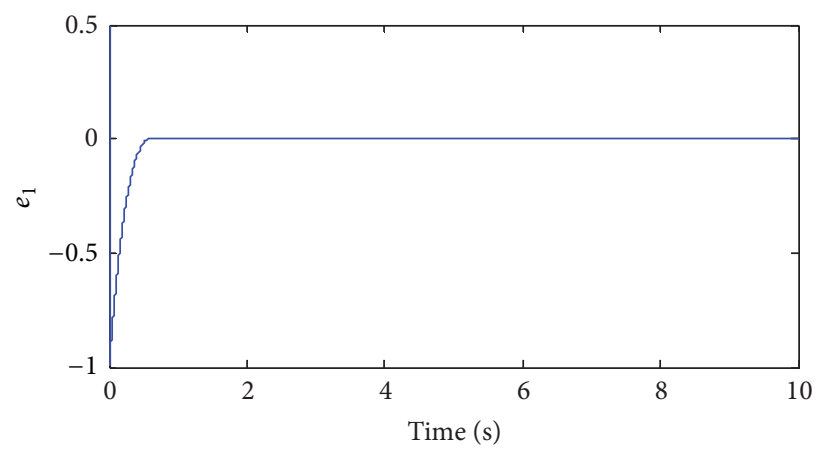

(a)

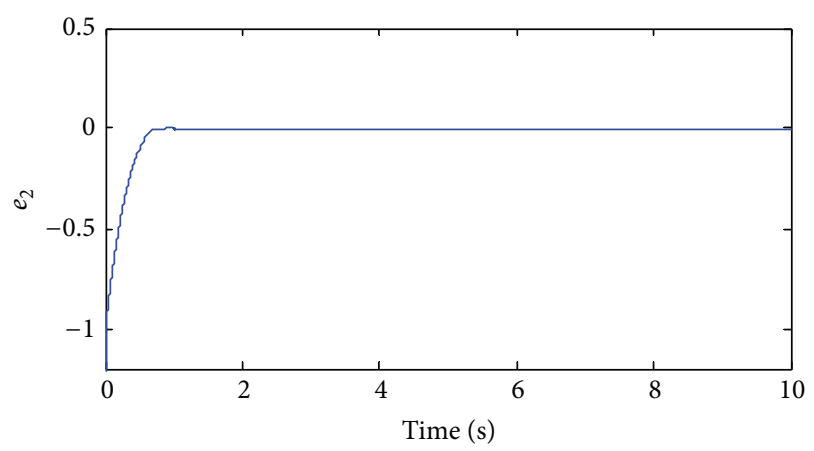

(b)

FIGURE 6: Synchronization errors of system (36) and system (37).

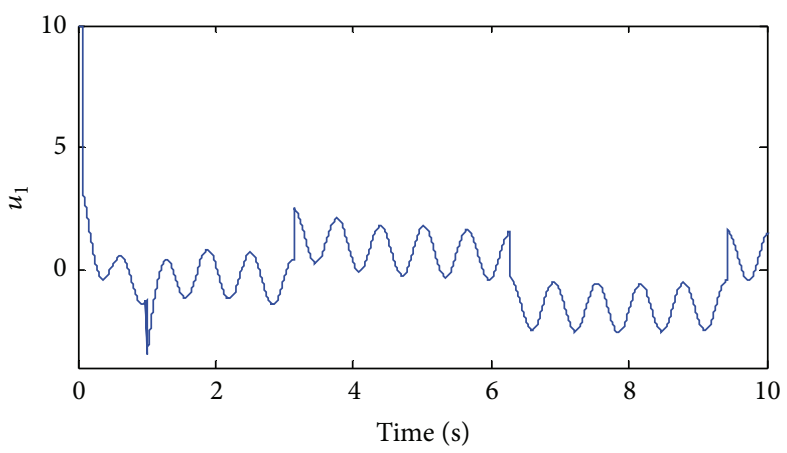

(a)

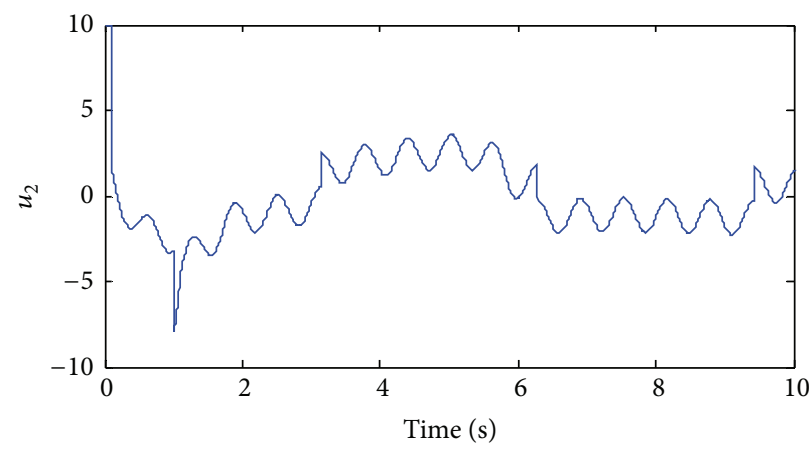

(b)

FIGURE 7: Control input of the slave system (37). 
$\tau(t)=0.1(\sin (0.5 t))+0.95$ in the master system. Figure 4 shows its dynamic behaviour with the initial condition $\left[x_{1}(t), x_{2}(t)\right]=[0.4,0.6](t \in[-1.05,0])$. Assume that the slave system cannot get the accurate parameters of the master system, so the slave system is given by (3) with the following parameters:

$$
\begin{gathered}
\widetilde{C}=\left[\begin{array}{ll}
1 & 0 \\
0 & 1
\end{array}\right], \quad \widetilde{A}=\left[\begin{array}{cc}
1 & -0.2 \\
-3 & 4.6
\end{array}\right], \\
\widetilde{B}=\left[\begin{array}{cc}
-2 & -0.1 \\
-0.1 & -3
\end{array}\right], \\
f(s)=\tanh (s), \quad \widetilde{\tau}(t)=1 .
\end{gathered}
$$

Assume that there is a nonlinear high frequency external disturbance $\mathbf{h}(t)=\cos 10 t+\operatorname{sign}(\sin t)$ in the communication channels. The observer and the controller are designed as in (14) and (13), and the parameters are given as follows:

$$
\begin{gathered}
\lambda=8, \quad \varepsilon=0.05, \quad \alpha=\frac{3}{7}, \\
k_{1}=4, \quad k_{2}=3 .
\end{gathered}
$$

Now we begin to simulate the synchronization for these two different systems on Matlab simulation platform. The initial condition associated with the slave system is $[-1,-1]$. The total simulation time is $10 \mathrm{~s}$, and the control signal begins to work at $0^{+} s$. Figure 5 shows the trajectories of the master system and the slave system, and the dynamics of synchronization errors are illustrated in Figure 6. The control inputs are shown in Figure 7. In addition, Figure 5 is magnified to show the synchronization performance clearly. It can be seen that the error system is stabilized in a short time and the slave system indeed synchronizes with the master system.

\section{Conclusions}

We have proposed an active finite-time synchronization method for uncertain chaotic neural networks. By using a differential observer, all the uncertainties have been estimated without requiring any prior information about the factors. The error dynamic system will become a singular perturbation system if $\varepsilon$ is small enough and we obtain its finitetime stability by analyzing two subsystems. Therefore, the slave system can synchronize with the master system even if there exist complex nonlinear disturbances and model uncertainties. As the method is simple to achieve and the control system shows strong robustness, it is believed that the results should provide some practical guidelines in practical engineering applications such as secure communication.

\section{Conflict of Interests}

The authors declare that there is no conflict of interests regarding the publication of this paper.

\section{Acknowledgments}

The authors would like to thank Beijing Higher Education Young Elite Teacher Project for their support and also all the reviewers for their constructive comments and suggestions to improve the quality of the paper.

\section{References}

[1] S. Boccaletti, J. Kurths, G. Osipov, D. L. Valladares, and C. S. Zhou, "The synchronization of chaotic systems," Physics Reports, vol. 366, no. 1-2, pp. 1-101, 2002.

[2] G. Kolumbán, M. P. Kennedy, and L. O. Chua, "The role of synchronization in digital communications using chaos. I. Fundamentals of digital communications," IEEE Transactions on Circuits and Systems I: Fundamental Theory and Applications, vol. 44, no. 10, pp. 927-936, 1997.

[3] L. M. Pecora and T. L. Carroll, "Synchronization in chaotic systems," Physical Review Letters, vol. 64, no. 8, pp. 821-824, 1990.

[4] J. Hu, S. Chen, and L. Chen, "Adaptive control for antisynchronization of Chua's chaotic system," Physics Letters A: General, Atomic and Solid State Physics, vol. 339, no. 6, pp. 455460, 2005.

[5] Q.-L. Han, "On designing time-varying delay feedback controllers for master-slave synchronization of Lur'e systems," IEEE Transactions on Circuits and Systems I: Regular Papers, vol. 54, no. 7, pp. 1573-1583, 2007.

[6] F.-H. Hsiao, "Delay-dependent exponential optimal $H^{\infty}$ synchronization for nonidentical chaotic systems via neuralnetwork-based approach," Abstract and Applied Analysis, vol. 2013, Article ID 294892, 16 pages, 2013.

[7] J.Sun, "Delay-dependent stability criteria for time-delay chaotic systems via time-delay feedback control," Chaos, Solitons \& Fractals, vol. 21, no. 1, pp. 143-150, 2004.

[8] M. Gupta, L. Jin, and N. Homma, Static and Dynamic Neural Networks: From Fundamentals to Advanced Theory, John Wiley and Sons, New Jersey, NJ, USA, 2004.

[9] F. Zou and J. A. Nossek, "Bifurcation and chaos in cellular neural networks," IEEE Transactions on Circuits and Systems I: Fundamental Theory and Applications, vol. 40, no. 3, pp. 166173, 1993.

[10] M. Gilli, "Strange attractors in delayed cellular neural networks," IEEE Transactions on Circuits and Systems I: Fundamental Theory and Applications, vol. 40, no. 11, pp. 849-853, 1993.

[11] T. Chen, W. Lu, and G. Chen, "Dynamical behaviors of a large class of general delayed neural networks," Neural Computation, vol. 17, no. 4, pp. 949-968, 2005.

[12] J. Zhou, T. Chen, and L. Xiang, "Robust synchronization of delayed neural networks based on adaptive control and parameters identification," Chaos, Solitons and Fractals, vol. 27, no. 4, pp. 905-913, 2006.

[13] C.-J. Cheng, T.-L. Liao, and C.-C. Hwang, "Exponential synchronization of a class of chaotic neural networks," Chaos, Solitons \& Fractals, vol. 24, no. 1, pp. 197-206, 2005.

[14] J. Cao and J. Lu, "Adaptive synchronization of neural networks with or without time-varying delay," Chaos, vol. 16, no. 1, Article ID 013133, 2006.

[15] B. Cui and X. Lou, "Synchronization of chaotic recurrent neural networks with time-varying delays using nonlinear feedback control," Chaos, Solitons and Fractals, vol. 39, no. 1, pp. 288-294, 2009. 
[16] H. Zhang, Y. Xie, Z. Wang, and C. Zheng, "Adaptive synchronization between two different chaotic neural networks with time delay," IEEE Transactions on Neural Networks, vol. 18, no. 6, pp. 1841-1845, 2007.

[17] W. He and J. Cao, "Adaptive synchronization of a class of chaotic neural networks with known or unknown parameters," Physics Letters A: General, Atomic and Solid State Physics, vol. 372, no. 4, pp. 408-416, 2008.

[18] Q. Gan, "Exponential synchronization of stochastic CohenGrossberg neural networks with mixed time-varying delays and reaction-diffusion via periodically intermittent control," Neural Networks, vol. 31, pp. 12-21, 2012.

[19] D. S. Goshi, K. M. K. H. Leong, and T. Itoh, "A secure highspeed retrodirective communication link," IEEE Transactions on Microwave Theory and Techniques, vol. 53, no. 11, pp. 35483556, 2005.

[20] B. Shen, Z. Wang, and X. Liu, "Sampled-data synchronization control of dynamical networks with stochastic sampling," IEEE Transactions on Automatic Control, vol. 57, no. 10, pp. 26442650, 2012.

[21] Z.-G. Wu and J. H. Park, "Synchronization of discrete-time neural networks with time delays subject to missing data," Neurocomputing, vol. 122, pp. 418-424, 2013.

[22] H. Dong, Z. Wang, and H. Gao, "Distributed $\mathrm{H}_{\infty}$ filtering for a class of markovian jump nonlinear time-delay systems over lossy sensor networks," IEEE Transactions on Industrial Electronics, vol. 60, no. 10, pp. 4665-4672, 2013.

[23] Z. Wang, H. Dong, B. Shen, and H. Gao, "Finite-horizon $H_{\infty}$ filtering with missing measurements and quantization effects," IEEE Transactions on Automatic Control, vol. 58, no. 7, pp. 1707$1718,2013$.

[24] Y. Liu, Z. Wang, J. Liang, and X. Liu, "Synchronization of coupled neutral-type neural networks with jumping-modedependent discrete and unbounded distributed delays," IEEE Transactions on Systems, Man, and Cybernetics B: Cybernetics, vol. 43, no. 1, pp. 102-114, 2012.

[25] Y. Sun and J. Cao, "Adaptive lag synchronization of unknown chaotic delayed neural networks with noise perturbation," Physics Letters A: General, Atomic and Solid State Physics, vol. 364, no. 3-4, pp. 277-285, 2007.

[26] Y. Sun, J. Cao, and Z. Wang, "Exponential synchronization of stochastic perturbed chaotic delayed neural networks," Neurocomputing, vol. 70, no. 13-15, pp. 2477-2485, 2007.

[27] S. C. Jeong, D. H. Ji, J. H. Park, and S. C. Won, "Adaptive synchronization for uncertain chaotic neural networks with mixed time delays using fuzzy disturbance observer," Applied Mathematics and Computation, vol. 219, no. 11, pp. 5984-5995, 2013.

[28] X. Li, C. Ding, and Q. Zhu, "Synchronization of stochastic perturbed chaotic neural networks with mixed delays," Journal of the Franklin Institute: Engineering and Applied Mathematics, vol. 347, no. 7, pp. 1266-1280, 2010.

[29] Q. Ma, S. Xu, Y. Zou, and G. Shi, "Synchronization of stochastic chaotic neural networks with reaction-diffusion terms," Nonlinear Dynamics, vol. 67, no. 3, pp. 2183-2196, 2011.

[30] V. Parra-Vega, S. Arimoto, Y.-H. Liu, G. Hirzinger, and P. Akella, "Dynamic sliding PID control for tracking of robot manipulators: theory and experiments," IEEE Transactions on Robotics and Automation, vol. 19, no. 6, pp. 967-976, 2003.

[31] J.-L. Zhao, J. Wang, and W. Wei, "Cascade control with active compensation for a class of uncertain nonlinear system," Journal of University of Science and Technology Beijing, vol. 34, no. 3, pp. 355-361, 2012 (Chinese).

[32] J. Alvarez-Ramírez, R. Suarez, and A. Morales, "Cascade control for a class of uncertain nonlinear systems: a backstepping approach," Chemical Engineering Science, vol. 55, no. 16, pp. 3209-3221, 2000.

[33] C. Zhang, J. Wang, Q. Guo, L. Zhao, and L. Fan, "Synchronization of uncertain perturbed chaotic neural network using tracking differential observer," in Proceeding of the International Symposium on Computer, Consumer and Control, Taichung, Taiwan, June 2014.

[34] H. K. Khalil and L. Praly, "High-gain observers in nonlinear feedback control," International Journal of Robust and Nonlinear Control, vol. 24, no. 6, pp. 993-1015, 2014.

[35] H. K. Khalil and J. W. Grizzle, Nonlinear Systems, vol. 3, Prentice hall, Upper Saddle River, NJ, USA, 2002.

[36] A. Bacciotti and L. Rosier, Liapunov Functions and Stability in Control Theory, Springer, London, UK, 2006.

[37] Y. Zhang and Q.-L. Han, "Network-based synchronization of delayed neural networks," IEEE Transactions on Circuits and Systems I: Regular Papers, vol. 60, no. 3, pp. 676-689, 2013. 


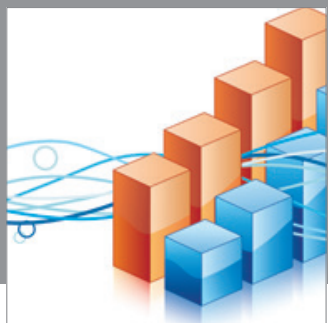

Advances in

Operations Research

mansans

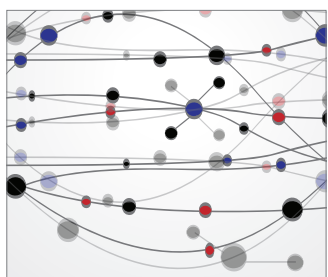

The Scientific World Journal
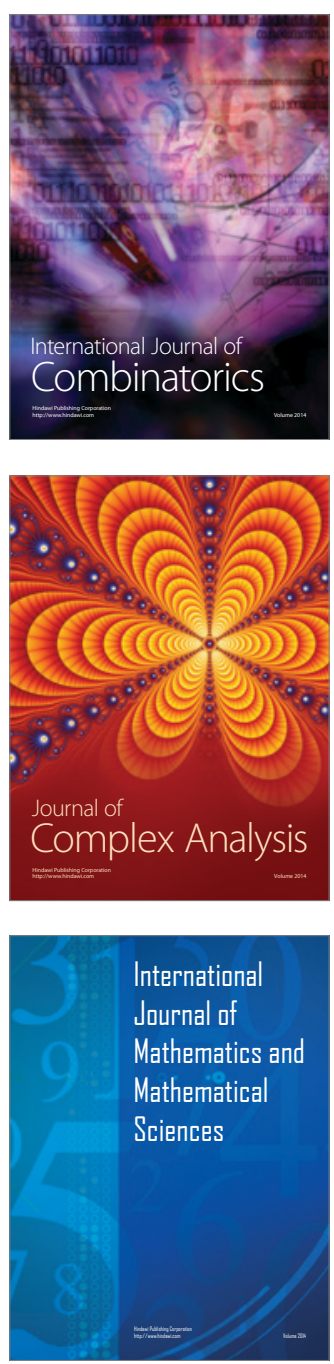
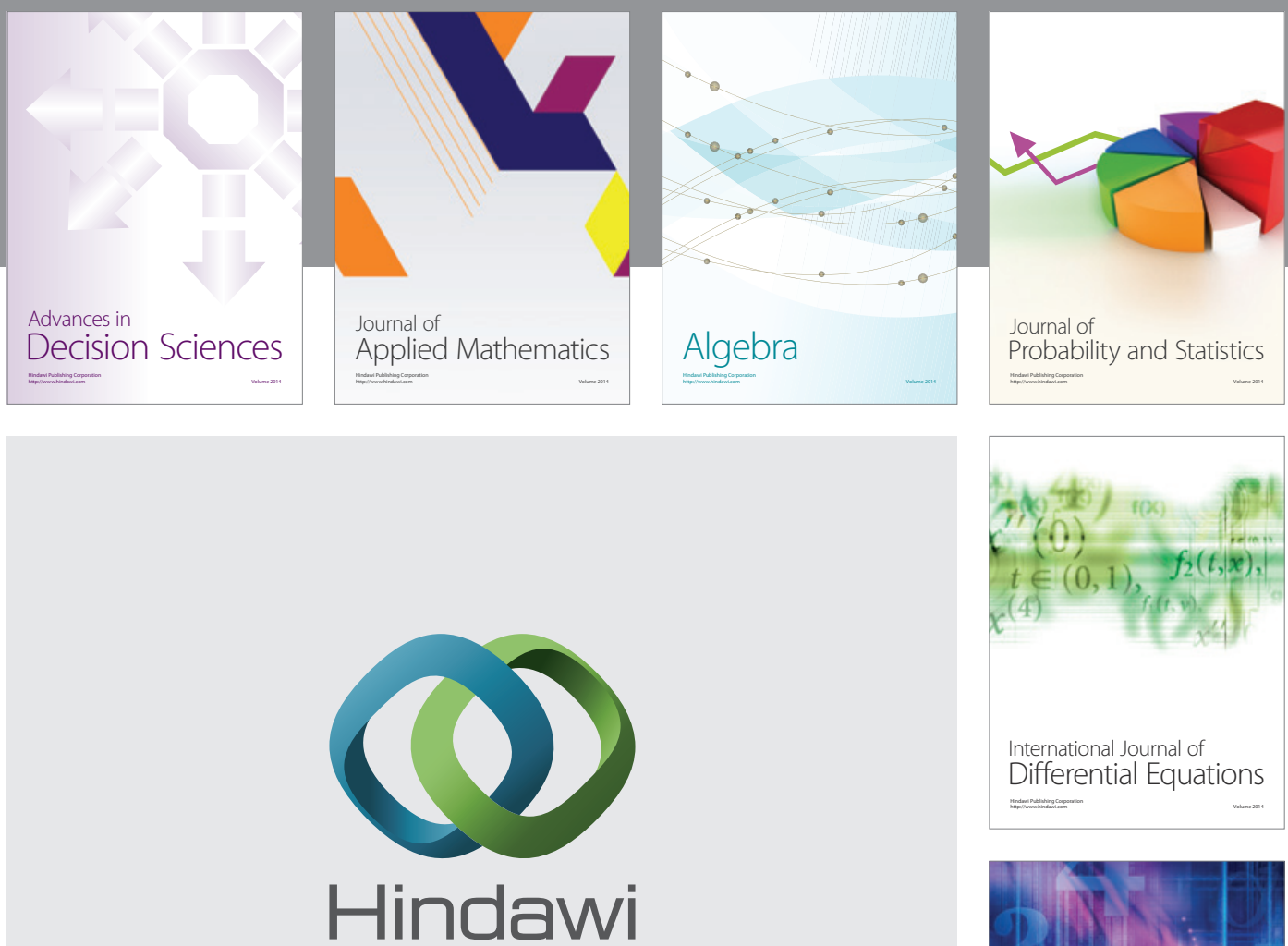

Submit your manuscripts at http://www.hindawi.com
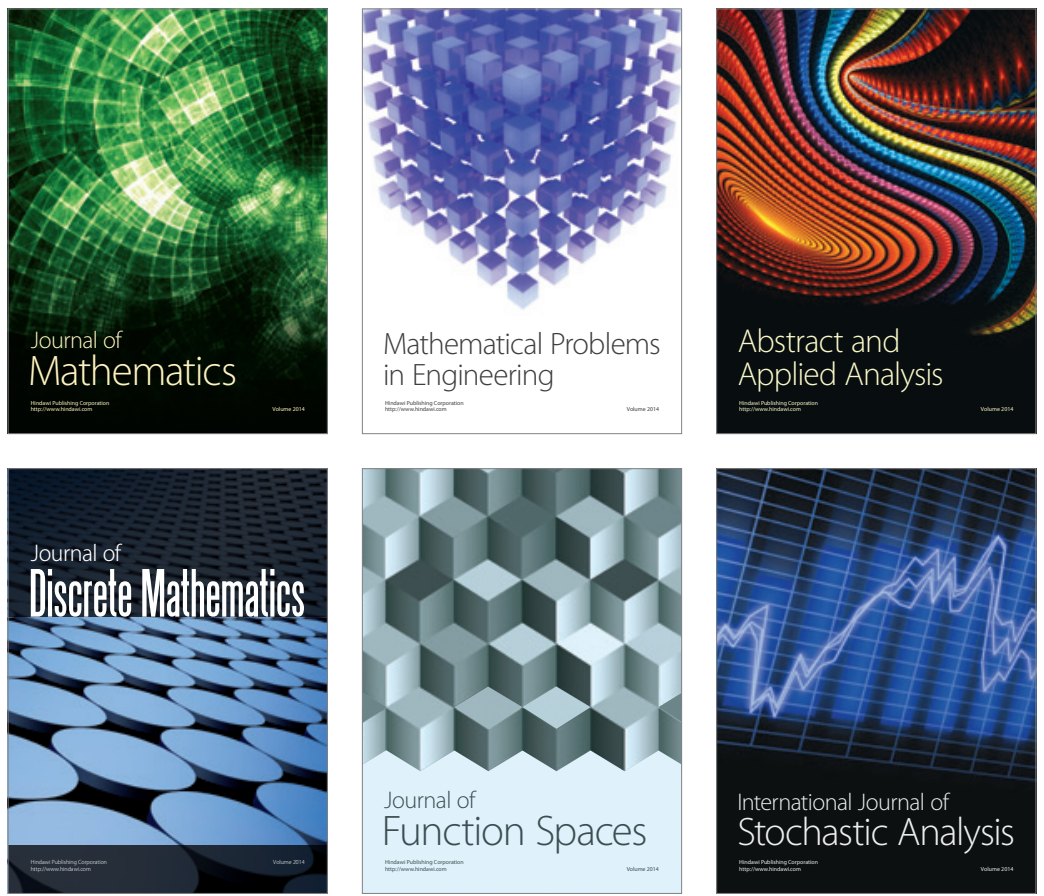

Journal of

Function Spaces

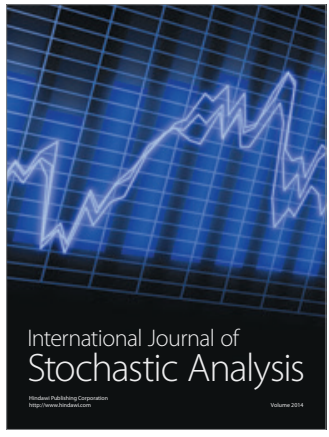

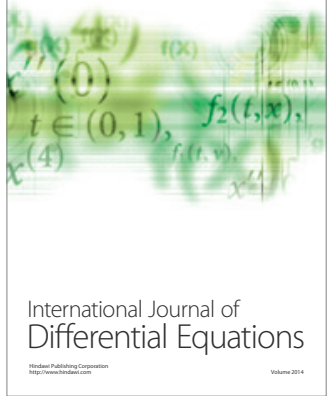
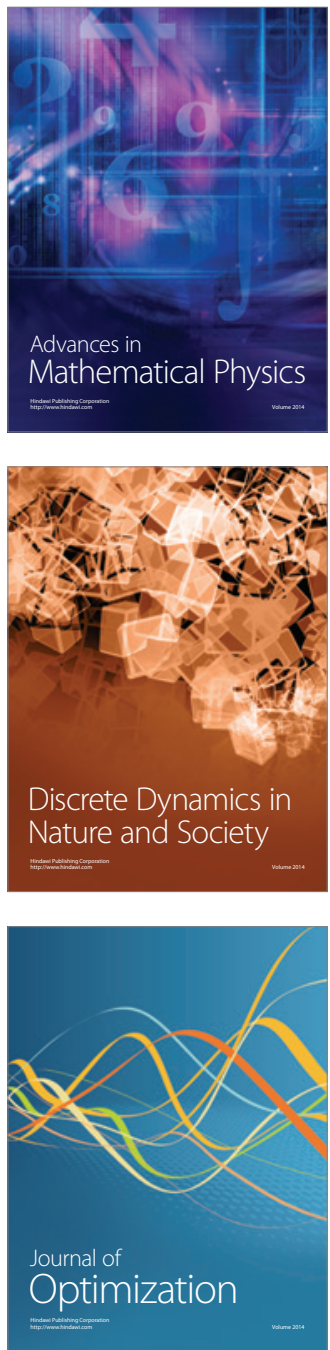